\title{
Erratum to: The generalized strong recurrence for non-zero rational parameters
}

\author{
TAKASHI NAKAMURA AND EUKASZ PAŃKOWSKI
}

\begin{abstract}
In the present paper, we prove that self-approximation of $\log \zeta(s)$ with $d=0$ is equivalent to the Riemann Hypothesis. Next, we show self-approximation of $\log \zeta(s)$ with respect to all nonzero real numbers $d$. Moreover, we partially filled a gap existing in "The strong recurrence for non-zero rational parameters" and prove self-approximation of $\zeta(s)$ for $0 \neq d=a / b$ with $|a-b| \neq 1$ and $\operatorname{gcd}(a, b)=1$.
\end{abstract}

Mathematics Subject Classification. Primary 11M06; Secondary 11M26.

Keywords. The Riemann zeta function, Self-approximation.

\section{Erratum to: Arch. Math. 95 (2010), 545-555 DOI 10.1007/s00013-010-0205-2}

1. Introduction. In 1982 Bagchi [1] discovered that the following almost periodicity holds in the critical strip if and only if the Riemann Hypothesis is true. To state it, let $\mu\{A\}$ stand for the Lebesgue measure of a measurable set $A, D:=\{s \in \mathbb{C}: 1 / 2<\operatorname{Re}(s)<1\}$, and let $H(K)$ denote the space of non-vanishing continuous functions on a compact set $K$, which are analytic in the interior, equipped with the supremum norm $\|\cdot\|_{K}$. Then Bagchi's result can be formulated as follows (see also [6, Section 8]).

T. Nakamura was partially supported by JSPS Grants 21740024 . Ł. Pańkowski was partially supported by the grant no. N N201 605940 from National Science Centre.

The online version of the original article can be found under doi:10.1007/s00013-010-0205-2. 
Theorem A. The Riemann Hypothesis holds if and only if, for every compact set $K \subset D$ with connected complement and for every $\varepsilon>0$, we have

$$
\liminf _{T \rightarrow \infty} \frac{1}{T} \mu\left\{\tau \in[0, T]:\|\zeta(s+i \tau)-\zeta(s)\|_{K}<\varepsilon\right\}>0 .
$$

In 2009 Nakamura [3] showed the following property which might be called self-approximation of the Riemann zeta function.

Theorem B. For every algebraic irrational number $d \in \mathbb{R}$, every compact set $K \subset D$ with connected complement, and every $\varepsilon>0$, we have

$$
\liminf _{T \rightarrow \infty} \frac{1}{T} \mu\left\{\tau \in[0, T]:\|\zeta(s+i d \tau)-\zeta(s+i \tau)\|_{K}<\varepsilon\right\}>0 .
$$

Note that the self-approximation with respect to almost all real numbers $d$ was also verified in [3]. Afterwards, Pańkowski [5] showed the above result for any irrational number $d$ whereas Garunkštis [2] and Nakamura [4] investigated the self-approximation for non-zero rational numbers, independently. Unfortunately, the papers $[2,4]$ contain a gap in the proof of the main theorem, so actually their methods work only for the logarithm of the Riemann zeta function (see Remark 2.3).

In this paper, we prove that self-approximation of $\log \zeta(s)$ with $d=0$ is equivalent to the Riemann Hypothesis in Theorem 2.1. Next, we show selfapproximation of $\log \zeta(s)$ for all nonzero real numbers $d$ in Theorem 2.2. Moreover, we partially filled the gap mentioned above and prove self-approximation of $\zeta(s)$ for $0 \neq d=a / b$ with $|a-b| \neq 1$ and $\operatorname{gcd}(a, b)=1$ in Theorem 3.1.

2. Self-approximation of $\log \zeta(s)$. Firstly, we show the following theorem which is an analogue of Theorem A.

Theorem 2.1. The Riemann Hypothesis holds if and only if, for every compact set $K \subset D$ with connected complement and for every $\varepsilon>0$, we have

$$
\liminf _{T \rightarrow \infty} \frac{1}{T} \mu\left\{\tau \in[0, T]:\|\log \zeta(s+i \tau)-\log \zeta(s)\|_{K}<\varepsilon\right\}>0 .
$$

Proof. If the Riemann hypothesis is true, we can apply Voronin's universality theorem.

Suppose that there exists a zero $\xi \in D$ of $\zeta(s)$. Put $K_{\varepsilon}:=\{s \in \mathbb{C}:|s-\xi| \leq \varepsilon\}$ $\subset D$. Now assume that for a neighborhood $K_{\varepsilon}$ of $\xi$ the following relation holds:

$$
\|\log \zeta(s+i \tau)-\log \zeta(s)\|_{K_{\varepsilon}}<\varepsilon .
$$

If a zero $\rho$ of $\zeta(s)$, where $\rho \in K_{\varepsilon}(\tau):=\{s \in \mathbb{C}:|s-\xi-i \tau| \leq \varepsilon\}$ does not exist, then the function $\log \zeta(s+i \tau)$ is analytic in the interior of $K_{\varepsilon}$ and bounded on $K_{\varepsilon}$. This contradicts to the above inequality. Hence a zero $\rho$ of $\zeta(s)$ exists in $K_{\varepsilon}(\tau)$. With regard to $(3)$ and the definition of $K_{\varepsilon}(\tau)$ the zeros $\xi$ and $\rho$ are intimately related; more precisely, $|\rho-\xi-i \tau|<\varepsilon$. Thus two different shifts $\tau_{1}$ and $\tau_{2}$ can lead to the same zero $\rho$, but their distance is bounded by $\left|\tau_{1}-\tau_{2}\right|<2 \varepsilon$. Therefore we obtain this lemma by modifying the proof of [6, Theorem 8.3] and using the classical Rouché theorem.

The reasoning of $[2$, Theorem $1 ; 5$, Theorem 1.1$]$ can be easily applied to prove self-approximation of $\log \zeta$. 
Theorem 2.2. For every real number $d \neq 0$, every compact set $K \subset D$ with connected complement, and for every $\varepsilon>0$, it holds that

$$
\liminf _{T \rightarrow \infty} \frac{1}{T} \mu\left\{\tau \in[0, T]:\|\log \zeta(s+i \tau)-\log \zeta(s+i d \tau)\|_{K}<\varepsilon\right\}>0 .
$$

Remark 2.3. In fact, in $[2,4]$ it was claimed that the above theorem holds even for the Riemann zeta function instead of the logarithm of $\zeta(s)$. Unfortunately, the proofs of the main results in [2, Theorem $1 ; 4$, Corollary 1.2] are not sufficient, since it was only shown that $|\zeta(s+d i \tau) / \zeta(s+i \tau)-1|<\varepsilon$. Obviously we have

$$
|\zeta(s+i \tau)-\zeta(s+i d \tau)|=|\zeta(s+i \tau)||\zeta(s+i d \tau) / \zeta(s+i \tau)-1| .
$$

So in order to prove self-approximation of $\zeta(s)$ it should have been proved that $\zeta(s+i \tau)$ is not too large, namely we need the inequality $|\zeta(s+i \tau)|<$ $|\zeta(s+i d \tau) / \zeta(s+i \tau)-1|^{-1}$, which is not necessarily satisfied.

3. Self-approximation of $\zeta(s)$. In the following theorem we partially fix the gap existing in $[2$, Theorem $1 ; 4$, Corollary 1.2$]$ and prove self-approximation of $\zeta(s)$ in the case $0 \neq d=a / b \in \mathbb{Q}$ with $|a-b| \neq 1$ and $\operatorname{gcd}(a, b)=1$.

Theorem 3.1. For every $0 \neq d=a / b$ with $|a-b| \neq 1$ and $\operatorname{gcd}(a, b)=1$, every compact set $K \subset D$ and for every $\varepsilon>0$, we have

$$
\liminf _{T \rightarrow \infty} \frac{1}{T} \mu\left\{\tau \in[0, T]:\|\zeta(s+i \tau)-\zeta(s+i d \tau)\|_{K}<\varepsilon\right\}>0 .
$$

Proof. First of all, note that it suffices to consider the case $a \neq b$, or equivalently $d \neq 1$.

Let us take $\omega\left(p_{m}\right):=\exp (2 \pi i m /(a-b))$, where $p_{m}$ denotes the $m$-th prime number. Then we have $\omega^{a}(p)=\omega^{b}(p)$ since $\omega^{a-b}(p)=1$. Firstly, we show that

$$
\left|\zeta_{z}\left(s, \omega^{c}\right)\right| \leq \exp \left(\frac{7\left(1-z^{1-2 \sigma}\right)}{2 \sigma-1}+|a-b|\left(z^{-\sigma}+2|s|\left(1-z^{-\sigma}\right)\right)\right),
$$

where $c=a, b$ and $\zeta_{z}\left(s, \omega^{c}\right):=\prod_{p \leq z}\left(1-\omega(p)^{c} p^{-s}\right)^{-1}$. In order to prove it let us consider the function $-\sum_{p \leq z} \log \left(1-\omega(p)^{c} p^{-s}\right)$. Then

$$
-\sum_{p \leq z} \log \left(1-\frac{\omega(p)^{c}}{p^{s}}\right)=\sum_{p \leq z} \sum_{k=1}^{\infty} \frac{\omega(p)^{c k}}{k p^{k s}}=\sum_{p \leq z} \frac{\omega(p)^{c}}{p^{s}}+\sum_{p \leq z} \sum_{2 \leq k} \frac{\omega(p)^{c k}}{k p^{k s}} .
$$

Let us estimate the latter sum on the right hand side. For $\sigma>1 / 2$ one has

$$
\begin{aligned}
\left|\sum_{p \leq z} \sum_{2 \leq k} \frac{\omega(p)^{c k}}{k p^{k s}}\right| & \leq \sum_{p \leq z} \sum_{2 \leq k} \frac{1}{p^{k \sigma}}=\sum_{p \leq z} \frac{1}{p^{2 \sigma}-p^{\sigma}} \leq 7 \sum_{p \leq z} \frac{1}{p^{2 \sigma}} \\
& \leq 7 \sum_{2 \leq n \leq z} \frac{1}{n^{2 \sigma}} \leq 7 \int_{1}^{z} t^{-2 \sigma} d t=\frac{7\left(1-z^{1-2 \sigma}\right)}{2 \sigma-1} .
\end{aligned}
$$

To consider the former sum, we put

$$
\omega(n):=\left\{\begin{array}{ll}
\omega(p) & \text { if } n=p \leq z, \\
0 & \text { otherwise, }
\end{array} \quad \Omega_{z}:=\sum_{n=1}^{z} \omega(n)^{c} .\right.
$$


Then, by partial summation, we obtain

$$
\begin{aligned}
\sum_{p \leq z} \frac{\omega(p)^{c}}{p^{s}} & =\sum_{n=1}^{z} \frac{\omega(n)^{c}}{n^{s}}=\frac{\Omega_{z}}{z^{s}}-\sum_{n=1}^{z-1} \Omega_{n}\left(\frac{1}{(n+1)^{s}}-\frac{1}{n^{s}}\right) \\
& =\frac{\Omega_{z}}{z^{s}}+s \sum_{n=1}^{z-1} \Omega_{n} \int_{n}^{n+1} t^{-s-1} d t .
\end{aligned}
$$

Let us notice that $\omega(p)^{c}$ is a nontrivial root of unity, since $\operatorname{gcd}(a, b)=1$ implies $a-b \nmid a, b$. Hence we have

$$
\begin{aligned}
\left|\sum_{p \leq z} \frac{\omega(p)^{c}}{p^{s}}\right| & \ll z^{-\sigma}+|s| \int_{1}^{z} t^{-\sigma-1} d t=z^{-\sigma}+\frac{|s|}{\sigma}\left(1-z^{-\sigma}\right) \\
& \leq \frac{1}{z^{\sigma}}+2|s|\left(1-\frac{1}{z^{\sigma}}\right),
\end{aligned}
$$

where the constant in symbol $\ll$ is equal to $|a-b|$. Therefore we obtain (6).

Now it suffices to follow the steps of the proof of [2, Theorem 1] and the following fact. Let $\|x\|$ give the the distance from a real number $x$ to the the nearest integer. Then the set of positive real numbers $\tau$ satisfying

$$
\max _{p_{m} \leq z}\left\|\tau \frac{\log p_{m}}{2 \pi}-\frac{m}{a-b}\right\|<\delta
$$

has a positive density for every positive $\delta$ by the Kronecker approximation theorem. Thus it holds for sufficiently large $z$

$$
\left\|\log \zeta(s+i c \tau)-\log \zeta_{z}\left(s, \omega^{c}\right)\right\|_{K}<\varepsilon, \quad c=a, b .
$$

This completes the proof.

\section{References}

[1] B. BagchI, A joint universality theorem for Dirichlet $L$-functions, Math. Z. 181 (1982), 319-334.

[2] R. Garunkštis, Self-approximation of Dirichlet $L$-functions, J. Number Theory 131 (2011), 1286-1295.

[3] T. NAKAmura, The joint universality and the generalized strong recurrence for Dirichlet $L$-functions, Acta Arith. 138 (2009), 357-362.

[4] T. NAKAmura, The generalized strong recurrence for non-zero rational parameters, Archiv der Mathematik 95 (2010), 549-555.

[5] Ł. PAŃkowski, Some remarks on the generalized strong recurrence for L-functions, New directions in value-distribution theory of zeta and $L$-functions, 305-315, Ber. Math., Shaker Verlag, Aachen, 2009.

[6] J. Steuding, Value-Distribution of L-functions, Lecture Notes in Mathematics, 1877, Springer, Berlin (2007). 
TAKASHI NAKAMURA

Department of Mathematics,

Faculty of Science and Technology,

Tokyo University of Science Noda,

Chiba 278-8510,

Japan

e-mail: nakamura_takashi@ma.noda.tus.ac.jp

ŁUKASZ PAŃKOWSKI

Faculty of Mathematics and Computer Science,

Adam Mickiewicz University,

Umultowska 87,

61-614 Poznan,

Poland

e-mail: lpan@amu.edu.pl

Received: 7 March 2012 